\title{
Koinfektion mit Streptococcus anginosus und Mycobacterium tuberculosis bei einem immunkompetenten pädiatrischen Patienten. Ein Fallbericht
}

\author{
Napoleon González-Saldaña ${ }^{a} \quad$ José Iván Castillo-Bejarano ${ }^{a} \quad$ Marte Hernández-Porras ${ }^{a}$ \\ Eduardo Arias de la Garza ${ }^{a}$ Sofia Fortes-Gutiérrez ${ }^{a}$ Jose Luis Copado-Gutiérrez ${ }^{a}$ \\ Hugo Juarez-Olguin ${ }^{b}$
}

${ }^{a}$ Abteilung für Infektionskrankheiten, National Institute of Pediatrics (NIP), Mexico City, Mexiko;

bPharmakologisches Labor, NIP und Institut für Pharmakologie, Medizinische Fakultät, Universidad Nacional Autónoma de Mexico, Mexico City, Mexiko

\section{Schlüsselwörter}

Tuberkulose · Streptococcus anginosus · Mycobacterium tuberculosis Pädiatrie $\cdot$ Thoraxabszess

\begin{abstract}
Hintergrund: Koinfektionen bei Tuberkulose (TB) sind selten. Eine Mischinfektion mit der Streptococcus-anginosus-Gruppe (SAG) und M. tuberculosis (MTB) wurde bei Kindern bislang noch nicht berichtet. Ziel dieses Berichts war die Beschreibung eines pädiatrischen Falls mit Lungenabszess infolge einer SAG-MTB-Doppelinfektion.

Fallvorstellung: Ein 11-jähriger Junge mit vor zwei Tagen akut aufgetretenen pulsierenden Schmerzen in der vorderen Brustwand. Der Patient berichtet über Fieber, Husten und Nasenlaufen in den vergangenen sieben Tagen. Die anteriore Thorax-Röntgenaufnahme ergab eine heterogene Verschattung am rechten Unterlappen,
\end{abstract}

und die laterale Projektion zeigte eine Obliteration an der vorderen Zwerchfellinsertion. Die parenterale Gabe von Ceftriaxon (100 mg/ kg/Tag) und Dicloxacillin (200 mg/kg/Tag) wurde eingeleitet. Der Abszess wurde anschließend drainiert und analysiert. Nach einjähriger Nachbeobachtung blieb der Patient weiterhin asymptomatisch.

Schlussfolgerung: Dies ist unseres Wissens der erste berichtete Fall einer pulmonalen Koinfektion mit MTB und SAG bei einem immunkompetenten pädiatrischen Patienten.

(C) 2020 The Author(s)

Published by S. Karger AG, Basel

\section{Hintergrund}

Das gleichzeitige Auftreten von Tuberkulose (TB) und einer bakteriellen Infektion ist eher unüblich. Es wurde eine Dreifachinfektion aus erworbenem Immundefizienzsyndrom, Mycobacterium tuberculosis (MTB) und Pneumokokken-Pneumonie berichtet, wobei es sich bei ersterem um einen maßgeblichen prädisponierenden Faktor für die Koinfektion mit MTB und anderen bakteriellen Erregern, hauptsächlich PneumokokkenPneumonie, handelt [1].

Die nach dem Erreger Streptococcus anginosus benannte AnginosusGruppe (SAG) gehört zur Kommensalflora der Mundhöhle und des
Urogenitaltrakts. Diese Erregergruppe verursacht charakteristische Infektionen mit Abszessbildung in verschiedenen Körperregionen [2]. Berichten zufolge ist die Prävalenz der Infektion bei männlichen Erwachsenen höher. Unseres Wissens wurde die Korrelation zwischen TB und SAG bei Kindern bislang jedoch noch nicht beschrieben. Es ist wichtig hervorzuheben, dass bei männlichen Patienten die Wahrscheinlichkeit von SAG-Koinfektionen aufgrund von x-chromosomalen primären Immundefizienzsyndromen wie der septischen Granulomatose erhöht sein könnte, obwohl diese sehr seltenen Infektionen nicht ausschließlich Männer betreffen.

Bei Erwachsenen sind die Prädilektionsstellen einer SAG-Infektion das Zentralnervensystem (ZNS), der Respirationstrakt und thor and the original publisher.
Hugo Juarez Olguin

Laboratorio de Farmacología, Instituto Nacional de Pediatría

Avenida Imán $N^{\circ} 1$, 3rd piso Colonia Cuicuilco

04530, Mexico City, Mexiko

juarezol@yahoo.co 
intraabdominelle Bereiche. In der pädiatrischen Population gehen SAG-Infektionen in der Regel mit einer Abszessbildung im Kopf, Hals und oberen Brustkorb einher [3].

Prädiktoren für eine Pneumonie bei Kindern sind Fieber und Zyanose plus zwei oder mehr der folgenden Anzeichen für Atemnot: Tachypnoe, Husten, Nasenflügeln, Hoover-Zeichen, Rasselgeräusche und verminderte Atemgeräusche. Für Kinder im Alter von über 5 Jahren definiert die Weltgesundheitsorganisation (World Health Organization, WHO) Tachypnoe als Atemfrequenz von mehr als 30 Atemzügen pro Minute.

SAG-Erreger finden sich verbreitet im Mund, im oberen Respirationstrakt, im Gastrointestinaltrakt und in Vaginalkulturen [4]. Die Rolle der SAG im Rahmen der Pathogenese von Atemwegsinfektionen wurde kürzlich von Mukae et al. basierend auf dem Ergebnis einer Auswertung von Klonbibliotheken beschrieben [5]. In einer Reihe handelte es sich bei einem Fünftel aller SAG-Infektionen um Thoraxinfektionen [4]. Gleichzeitige Infektionen mit MTB und anderen Erregern sind selten, treten meist unter Beteiligung von S. pneumoniae auf und werden am häufigsten bei $\mathrm{Pa}-$ tienten mit HIV-Infektion berichtet [1]. Der Nachweis einer SAGKoinfektion mit MTB wurde bei pädiatrischen Patienten bislang klinisch nicht beschrieben [6]. Im vorliegenden Fallbericht schildern wir eine Abszessbildung verursacht durch eine SAG-MTBDoppelinfektion bei einem immunkompetenten pädiatrischen Patienten. Die ungewöhnliche Entwicklung des TB-Bildes bei einem pädiatrischen Patienten macht diesen Fallbericht besonders bemerkenswert. Innerhalb weniger Tage entwickelte sich bei dem Patienten ein Lungenabszess. Als einziger Krankheitserreger wurde bei der automatisierten Analyse mit dem BD-Phoenix-System SAG identifiziert, ein eher seltener Befund.

\section{Fallvorstellung}

Ein 11-jähriger Junge, wohnhaft in Mexico City und bis dahin gesund, Gewicht $42 \mathrm{~kg}$ und 1,32 m groß, wurde mit vor zwei Tagen akut aufgetretenen pulsierenden Schmerzen in der vorderen Brustwand in die Notaufnahme des Instituto Nacional de Pediatría, Mexico City, aufgenommen. Der Patient berichtete über Fieber, Husten und Nasenlaufen in den vergangenen sieben Tagen. Auf Nachfrage gaben die Eltern keine konstitutionellen Symptome wie Gewichtsverlust, Asthenie, Adynamie oder nächtliches Schwitzen an. Der Impfpass des Kindes war gemäß den nationalen Empfehlungen komplett und beinhaltete eine BCG-Impfung bei der Geburt. Eine Untersuchung der familiären Kontakte durch die epidemiologische Abteilung, die sich auf die gesamte Familie (Geschwister, Eltern und Großeltern) sowie die Nachbarschaft erstreckte, war negativ und ergab in der Vorgeschichte keine TB oder anderen wichtigen Komorbiditäten in der Familie oder bei engen Kontaktpersonen. Eine erweiterte Untersuchung an der Schule wurde durch die epidemiologische Abteilung nicht durchgeführt. Ein bei der Aufnahme des Patienten in der Notaufnahme angefertigtes Elektrokardiogramm (EKG) zeigte einen Rechtsschenkelblock, der als physiologisch eingestuft wurde. Ein Thorax-Röntgen ergab keine bedeutsamen Befunde, und der Pa- tient wurde daraufhin entlassen. Einen Tag nach seiner Entlassung wurde der Patient erneut wegen Schmerzen in der Brust, nun in der rechten Seite des Brustkorbs lokalisiert, mit Dyspnoe und $38,6^{\circ} \mathrm{C}$ Fieber aufgenommen.

\section{Diagnose und Behandlung}

Bei seiner erneuten Aufnahme zeigte der Patient Tachypnoe mit beidseitigen Retraktionen des oberen und unteren Brustkorbs ohne Anzeichen einer Hypoxie. Ein großes Blutbild (cell blood count, CBC) ergab einen leichten Anstieg $\left(14.000 / \mathrm{mm}^{3}\right)$ mit signifikant hoher absoluter Neutrophilenzahl (absolute neutrophil count, ANC) (13.440/ $\mathrm{mm}^{3}$ Neutrophile). Es wurde ein anteriores ThoraxRöntgen (Abb. 1a, b) durchgeführt, das eine heterogene Verschattung am rechten Unterlappen ergab, und die laterale Projektion zeigte eine Obliteration an der vorderen Zwerchfellinsertion.

Es wurde eine komplizierte Pneumonie diagnostiziert, und die parenterale Gabe von Ceftriaxon (100 mg/kg/Tag) und Dicloxacillin $(200 \mathrm{mg} / \mathrm{kg} / \mathrm{Tag})$ wurde eingeleitet. Dies ist an unserem Krankenhaus die Behandlung der Wahl zur Bekämpfung gramnegativer Bakterien und Staphylokokken, wie sie häufig zur Behandlung von Patienten mit schwerer und komplizierter Pneumonie angewendet wird. Wir wenden dieses Schema hauptsächlich zur Behandlung von Infektionen mit S. pneumoniae und $S$. aureus und stets nach Maßgabe der Befunde klinischer und ergänzender Untersuchungen wie Röntgen, Blutbild und PCR an. Da sich der Zustand des Patienten jedoch weniger verbesserte als erwartet, führten wir eine Ultraschalluntersuchung des Thorax durch. Diese ergab ein septiertes Empyem von etwa $260 \mathrm{ml}$ mit Atelektase der rechten Basis. Es wurde ein Thoraxkatheter gelegt, über den eine trübe Flüssigkeit mit 375 Zellen (80\% Neutrophile, 8\% Lymphozyten, 9\% Monozyten und 3\% Eosinophile), einem $\mathrm{pH}-$ Wert von 8, 5,31 g/dl Proteinen und $24 \mathrm{mg} / \mathrm{dl}$ Glukose drainiert wurde. Auf die Bestimmung von LDL und Triglyzeriden wurde verzichtet, da es sich nicht um Routinetests handelt und sie für den vorliegenden Fall als irrelevant betrachtet wurden. Der Thoraxkatheter wurde nach fünftägiger Drainage entfernt. Trotz Flüssigkeitsdrainage und Antibiose blieb der Patient weiterhin febril und wurde auf parenteral verabreichtes Vancomycin umgestellt, da eine mögliche Infektion mit Methicillin-resistentem Staphylococcus aureus (MRSA) oder Cephalosporin-resistentem Streptococcus pneumoniae vermutet wurde. Dreizehn Tage nach der erneuten Aufnahme kam es bei dem Patienten zu einer Hämoptyse-Episode und einem Anstieg des C-reaktiven Proteins (CRP) (von 8,4 mg/dl auf 12,7 mg/dl). Weitere Untersuchungen wegen des persistierenden Fiebers wurden durchgeführt, u.a. eine Untersuchung auf MTB, da der Patient aus einer Gegend mit einer jährlichen TB-Inzidenz von 9,1/100.000 kam, auch wenn kein betroffener Haushalt oder entsprechende enge Kontakte dokumentiert waren. Eine Sputumprobe wurde mit Hilfe des Gentests Xpert MTB/Rifampicin (RIF) analysiert und erwies sich als positiv für MTB-Komplex und negativ für rpoB-Mutationen. Zu einem späteren Zeitpunkt wurde wirkstoffempfindliches MTB in einer Mykobakterienkultur isoliert. Bei Durchführung einer 


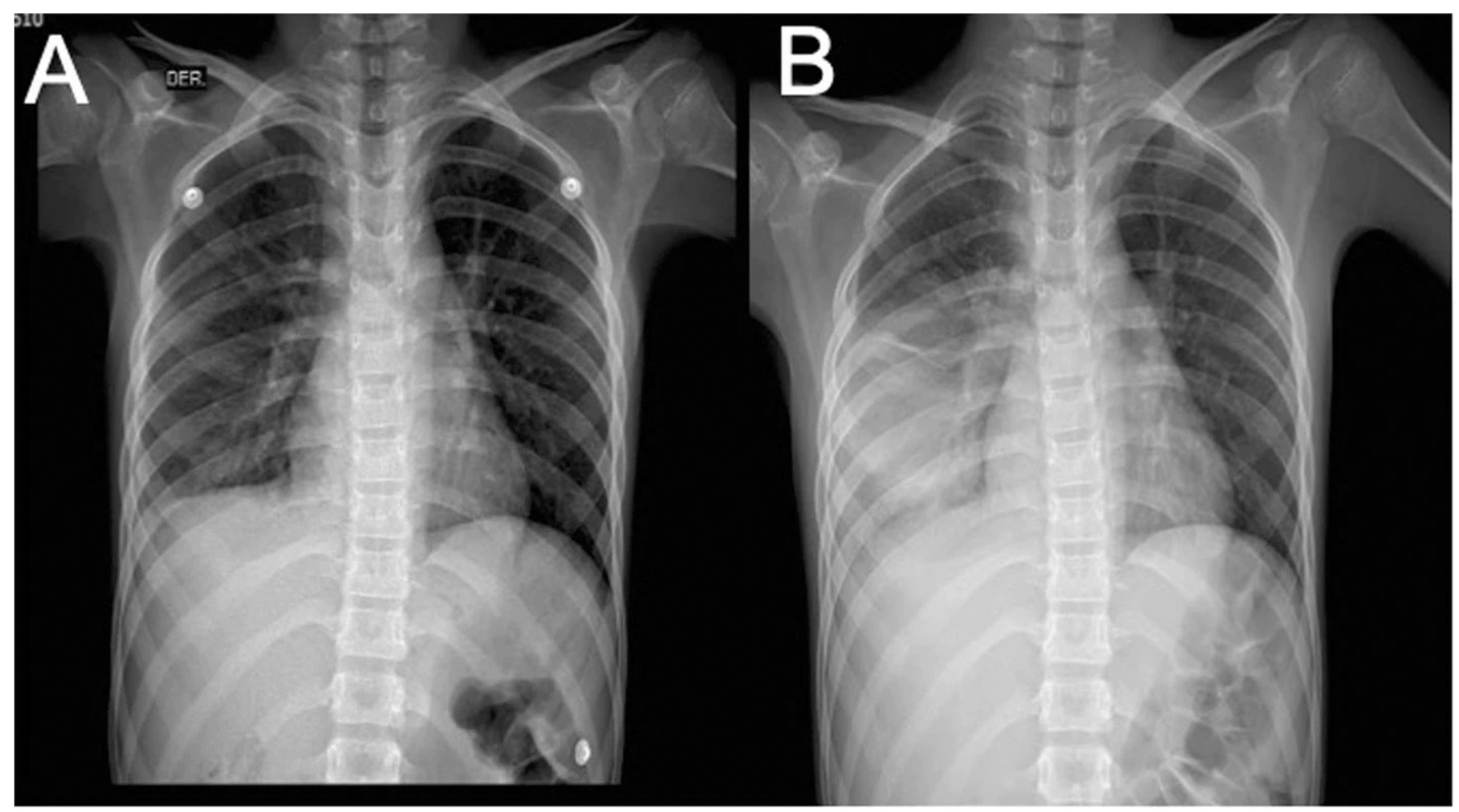

Abb. 1. (a) Bei Aufnahme angefertigtes Röntgenbild mit Vorliegen eines Pleuraergusses rechts. (b) Konsolidierung an der rechten Basis und Pleuraerguss.

Thorax-Computertomographie ergab sich ein Bild, das mit einem Lungenabszess von 75,7 mm x 49,18 mm kompatibel war (Abb. 2). Eine Lungen-Computertomographie bestätigte die TB-Diagnose. Der Abszess wurde anschließend drainiert und analysiert. Das aus dem Abszess drainierte eitrige Sekret wurde über 24 Stunden in Blutagar inkubiert und ließ das Wachstum kleiner Kolonien mit $\alpha$-Hämolyse erkennen. In einem BD-PhoenixTM-Test wurde anschließend SAG identifiziert. Eine aufgrund des Verdachts auf eine Koinfektion bei Aufnahme des Patienten durchgeführte Untersuchung des eitrigen Sekrets auf aerobe und anaerobe Erreger, Pilze und Mykobakterien sowie der Gentest mittels Xpert MTB/ RIF (Cepheid Inc., CA, USA) und ein Ausstrich säurefester Bazillen ergaben ausschließlich SAG.

Nach kontrollierter Drainage wurden neue Röntgenaufnahmen angefertigt, und das klinische Ergebnis des Patienten war günstig. Das Fieber sank nach der Lymphdrainage innerhalb von etwa 4 Tagen. Eine antituberkulöse Therapie wurde eingeleitet (INH/ RIF/PZA/E), und die Behandlung mit Vancomycin wurde über 28 Tage fortgesetzt. Ein Panel für Immundefekte ergab eine normale Nitrotetrazoliumblau- und Immunglobulin-Quantifizierung, während der IL-12-/gamma-Interferon-Test und ein HIVELISA der 4. Generation negativ waren. Der Patient wurde nach 37-tägigem stationären Aufenthalt bei adäquatem Ansprechen auf die Behandlung entlassen. Das Kind schloss eine zweimonatige intensive Anti-TB-Behandlung ab und wurde später auf eine viermonatige Erhaltungstherapie mit INH/RIF umgestellt. Nach einjähriger Nachbeobachtung blieb der Patient weiterhin asymptomatisch.

\section{Diskussion}

TB-Abszesse sind bei immunkompetenten Wirten selten, und in den meisten Fällen wird eine Beteiligung der Wirbelsäule berichtet [7]. Doppelinfektionen mit TB und Bakterien wurden bereits früher beschrieben. Allerdings treten sie bei immunkompetenten Patienten eher selten auf. Bei Patienten mit HIV-Infektion sind sie sehr viel häufiger, da deren Risiko für eine Infektion mit Pneumokokken (dem häufigsten Koinfektionserreger) höher ist [6].

Bei SAG-Bakterien handelt es sich um fakultativ anaerobe Erreger, die die Mundhöhle und die oberen Atemwege besiedeln. Infektionen mit SAG zeichnen sich in der Regel durch Abszessbildung und Pleuraempyem aus. Gemäß Okada et al. [8] sind an 54,5\% aller polymikrobiellen Infektionen SAG-Erreger beteiligt, und bei 66,7\% dieser Population ist gewöhnlich eine frühzeitige chirurgische Behandlung erforderlich. Die Sterblichkeit ist niedrig, und aus dem eitrigen Sekret des drainierten Empyems oder Abszesses lässt sich kein MTB isolieren. In einer weiteren Studie berichten Noguchi et al. [4] eine Koinfektionsrate von 20\%, u.a. mit $H$. influenzae, Bacteroides spp. und anderen Erregern bei einer Sterblichkeitsrate von 6,7\%.

Der im vorliegenden Fall verfolgte Behandlungsansatz bestand in einer chirurgischen Drainage des Abszesses ohne Dekortikation und Antibiotikatherapie über 35 Tage. Auch wenn SAG-Infektionen bei Patienten mit TB-Koinfektion in der Regel eine akute oder subakute Erkrankung verursachen, ist aus klinischer Sicht unter Umständen nicht leicht auszumachen, wie weit die jeweiligen Erreger zum Krankheitsbild beitragen. Berichten zufolge können ViridansStreptokokken das Wachstum von MTB in vitro hemmen; es ist 


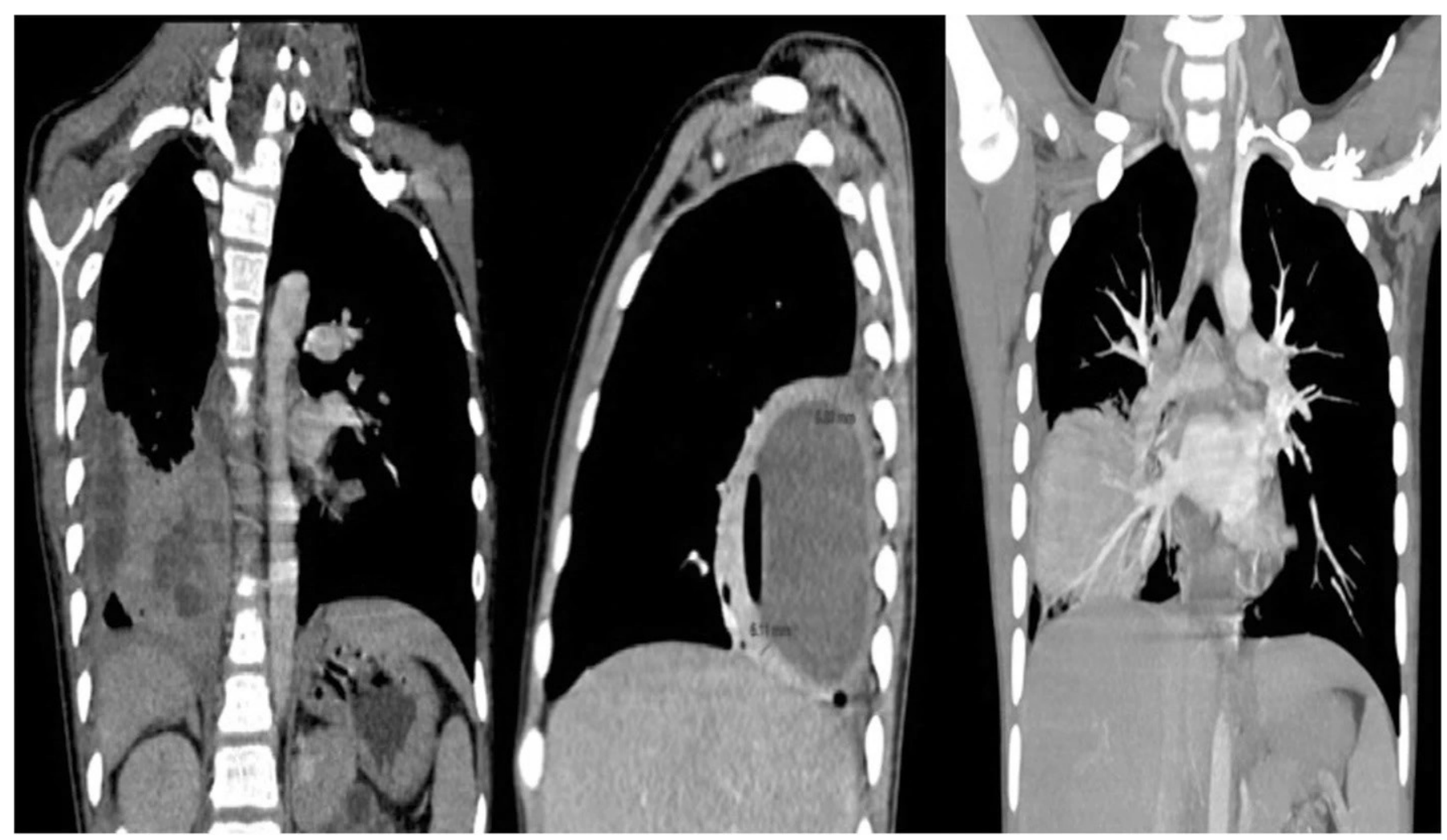

Abb. 2. Kavitäre Pneumonie (Abszess) im Segmentum basale mediale, posterius und laterale rechts, assoziiert mit einer Adenopathie der mediastinalen Lymphknoten.

jedoch weiterhin unklar, ob MTB in der Lage ist, die Pathogenität von SAG bei Vorliegen beider Erreger im Rahmen derselben Infektion zu verstärken $[9,10]$. In unserem Fall zeigte die Erkrankung bei vorliegender Lungen-TB einen raschen Ausbruch mit Pleuraerguss und Abszessbildung. Aus dem eitrigen Sekret des drainierten Pleuraergusses und Abszesses wurde in aerober Bakterienkultur SAG isoliert. Die übrigen Kulturen waren negativ. Das akute Auftreten einer ambulant erworbenen Pneumonie wurde hauptsächlich an Orten mit hoher TB- und HIV-Inzidenz beschrieben [11].

Primäre Immundefizienzsyndrome wie septische Granulomatose, eine Suszeptibilität nach den Mendel'schen Regeln für mykobakterielle Erkrankungen, schwere kombinierte Immundefekte (severe combined immunodeficiency, SCID) und eine sekundäre HIV-Immunität sind u.a. als Risikofaktoren für eine TB-Erkrankung bekannt [12]. Männliches Geschlecht, parodontale Erkrankungen, Alkoholabusus, Krebserkrankungen, HIV-Infektion und zystische Fibrose sind allesamt prädisponierende Faktoren für eine SAG-Infektion [13]. Unseres Wissens wurde eine Korrelation zwischen TB und SAG bislang noch nicht beschrieben. Unser Patient wurde entsprechend der WHO-Empfehlung zur Behandlung der wirkstoffempfindlichen Tuberkulose mit einem 2HRZE/4HR-Regime behandelt [14].

\section{Schlussfolgerung}

Dies ist unseres Wissens der erste berichtete Fall einer pulmonalen Koinfektion mit MTB und SAG bei einem immunkompeten- ten pädiatrischen Patienten. In Anbetracht der maßgeblichen Implikationen in Bezug auf die klinische Behandlung und das Behandlungsergebnis sollten sowohl häufige (z.B. S. pneumoniae) als auch unübliche Erreger (wie SAG) als potenzielle koinfizierende Pathogene in Betracht gezogen werden, insbesondere in Situationen oder Regionen mit hoher TB-Belastung.

\section{Zustimmung zur Veröffentlichung}

Von den Eltern des Patienten wurde eine schriftliche Einverständniserklärung für die Veröffentlichung des vorliegenden Fallberichts und der dazugehörigen Bilder eingeholt.

\section{Disclosure Statement}

Die Autoren erklären, dass keine Interessenskonflikte bestehen.

\section{Literatur}

Die Literatur ist unter www.karger.com/Article/Fulltext/508635 abrufbar.

\section{Lizenzangabe}

González Saldaña N, Castillo Bejarano JI, Hernández Porras M, Arias de la Garza E, Fortes Gutiérrez S, Copado Gutiérrez JL, Juarez Olguin H: Coinfection with Streptococcus anginosus and Mycobacterium tuberculosis in an immunocompetent pediatric patient. A case report. BMC Pulm Med. 2020;20(1):5 (DOI: 10.1186/s12890-019-1044-y). @ 2020 The Author(s). (Übersetzung; Verfügbarkeit der Daten und Materialien, Beiträge der Autoren, Danksagungen, Finanzielle Unterstützungen, Ethikerklärungen, Abkürzungen und Publisher's Note gekürzt), lizensiert unter CC BY 4.0 (https://creativecommons.org/licenses/by/4.0/deed.de). 